\title{
Correction to: CR Sub-Laplacian Comparison and Liouville-Type Theorem in a Complete Noncompact Sasakian Manifold
}

Shu-Cheng Chang ${ }^{1,2} \cdot$ Ting-Jung Kuo ${ }^{3} \cdot$ Chien Lin $^{4} \cdot$ Jingzhi Tie $^{5}$ (D)

Published online: 1 August 2018

(c) Mathematica Josephina, Inc. 2018

\section{Correction to: The Journal of Geometric Analysis https://doi.org/10.1007/s12220-018-0056-9}

The original version of this article was updated to correct Eq. 3.7.

The original article can be found online at https://doi.org/10.1007/s12220-018-0056-9.

$\bowtie$ Jingzhi Tie

jtie@math.uga.edu

Shu-Cheng Chang

scchang@math.ntu.edu.tw

Ting-Jung Kuo

tjkuo1215@ntnu.edu.tw

Chien Lin

r97221009@ntu.edu.tw

1 Department of Mathematics and Taida Institute for Mathematical Sciences (TIMS), National Taiwan University, Taipei 10617, Taiwan

2 Present Address: Yau Mathematical Sciences Center, Tsinghua University, Beijing, China

3 Department of Mathematics, National Taiwan Normal University, Taipei 11677, Taiwan

4 Department of Mathematics, National Tsing Hua University, Hsinchu 30013, Taiwan

5 Department of Mathematics, University of Georgia, Athens, GA 30602-7403, USA 